\title{
Editorial
}

\section{Dynamics of Multibody Systems in Research and Education}

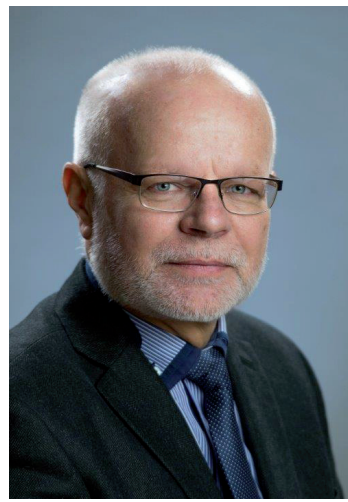

\author{
prof. Ing. Štefan Seglá, CSc.
}

Technical University of Košice, Faculty of Mechanical Engineering, Department of Applied Mechanics and Mechanical Engineering

Štefan Segla, prof., Ing., CSC., (born in 1954) is a professor at the Technical University of Košice, Faculty of Mechanical Engineering, Institute of Design and Process Engineering. He graduated from the Faculty of Mechanical Engineering, (zech Technical University in Prague in 1978. His research areas are kinematic and dynamic analysis and optimization of mechanical and mechatronical systems. He is an author of three monographs, twelve textbooks and more than one hundred scientific publications in journals and conference proceedings in Slovakia and abroad. Forteen papers are registered in Web of Science and SCOPUS. He is a member of IFTOMM (International Federation for the Promotion of Mechanism and Machine Science), its Permanent Commission for the Standardization of Terminology and Multibody Dynamics, a vice-chairman of the Slovak National Committee of IFToMM, a member of the (zech Society of Mechanics, Slovak Society of Mechanics and Central European Association for Computational Mechanics.

Many common mechanisms and machines such as vehicle and aircraft subsystems, robotic systems, biomechanical and mechatronic systems consist of rigid and deformable bodies that undergo large rigid body motion. The bodies are interconnected with kinematical constraints and coupling elements. The dynamics of these large-scale and nonlinear systems under dynamic loading in most cases can only be solved with methods of computational mechanics. For these systems the concept of the multibody dynamics can be successfuly utilized. Their mechanical models should be as complex as necessary but also as simple as possible. Mechanical modelling is an iterative process that needs a lot of engineering experience. Both viscoelastic and inertia properties of technical systems are discretized during the process of shaping of the system's mechanical model. This model has to be able to describe mechanical properties of the real system which influence the phenomena under consideration.

Many technical systems consist of a large number of bodies interconnected with the constraint elements such as joints, bearings, springs, dampers and actuators. It can be stated generally that if the bodies in a system undergo large rigid body motion and small elastic vibrations, modelling using the multibody system approach is a very powerful tool. Another part of the mechanical modelling is the idealized description of the real load. It may be introduced in the model as concentrated forces and moments as well as forces and moments distributed over line, surface and volume.

Once the mechanical model is built, in the second step its mathematical model (a set of governing equations) has to be formulated. The mathematical modelling of the established 
mechanical model leads to one of the two forms of the governing equations (GE)

- Descriptor form - the number of coordinates and GE are larger than the number of degrees of freedom (DOF). The type of the GE is differential algebraic equations. The degree of nonlinearity of the GE is low.

Minimal form - the number of coordinates and GE is equal to the number of DOF. The type of the GE is the ordinary differential equations. The degree of nonlinearity of the GE is high.

A good and effective mathematical model has to reflect the type and character of the analysis that is to be performed (e.g. linear and nonlinear analysis). Nowadays numerous computational techniques and algorithms of generating GE for various classes of problems are at the user's disposal. These algorithms are the core of various commercial program packages. An appropriate mathematical modelling can significantly influence the computational procedure itself to a great extent - reducing the computational time and/or gaining more accurate results.

In multibody dynamics various classes of problems can be distinguished. The most important are as follows

- Forward dynamics - determination of the motion of a system that is subjected to the prescribed applied forces.

- Inverse dynamics - determination of the applied and constraint forces for a mechanical system whose motion is prescribed. Within the framework of the quasi-static approach, the inertia forces of the system are neglected.

- Vibrations - in most of the cases in linear domain, solving the vibrational problem leads to the determination of the system eigenvalues and modes. In this context the system stability problem can also be mentioned.

Multibody systems consist of elements (bodies) with inertia and coupling elements without inertia. Within the coupling elements two types of actuators can be distinguished

- Force actuators - applied forces are prescribed in the form of time functions (forward dynamic analysis).

- Displacement actuators - displacements of the system's bodies are prescribed in the form of time functions (inverse dynamic analysis). The actuators of this type can be considered as kinematical constraints.

The problem of optimization of mechanical systems is of great importance in engineering. Among often optimized quantities can be named weight, cost, various dynamic characteristics of the system etc. Optimization can be defined as the process of finding conditions that gives the maximum or minimum value of a goal function. Optimization methods and algorithms coupled with modern tools of computeraided design, are being used to enhance the process of conceptual and also detailed design of engineering systems. Optimization, in its broadest sense, can be applied to solve any engineering problem.

Figure below illustrates modelling and optimization of two types of suspension systems (passive and semiactive suspension) of a passenger car modelled in the general multibody program CarSim. Its open source capability enabled replacement the passive dampers by dry friction semiactive dampers modelled in MATLAB/Simulink. The control strategy based on reduced feedback was modified to avoid chatter and sticking. The results of optimization showed that the semiactive friction dampers can significantly improve not only the ride comfort but also handling properties (dynamic tyre forces) of passenger cars compared with the passive suspension systems.

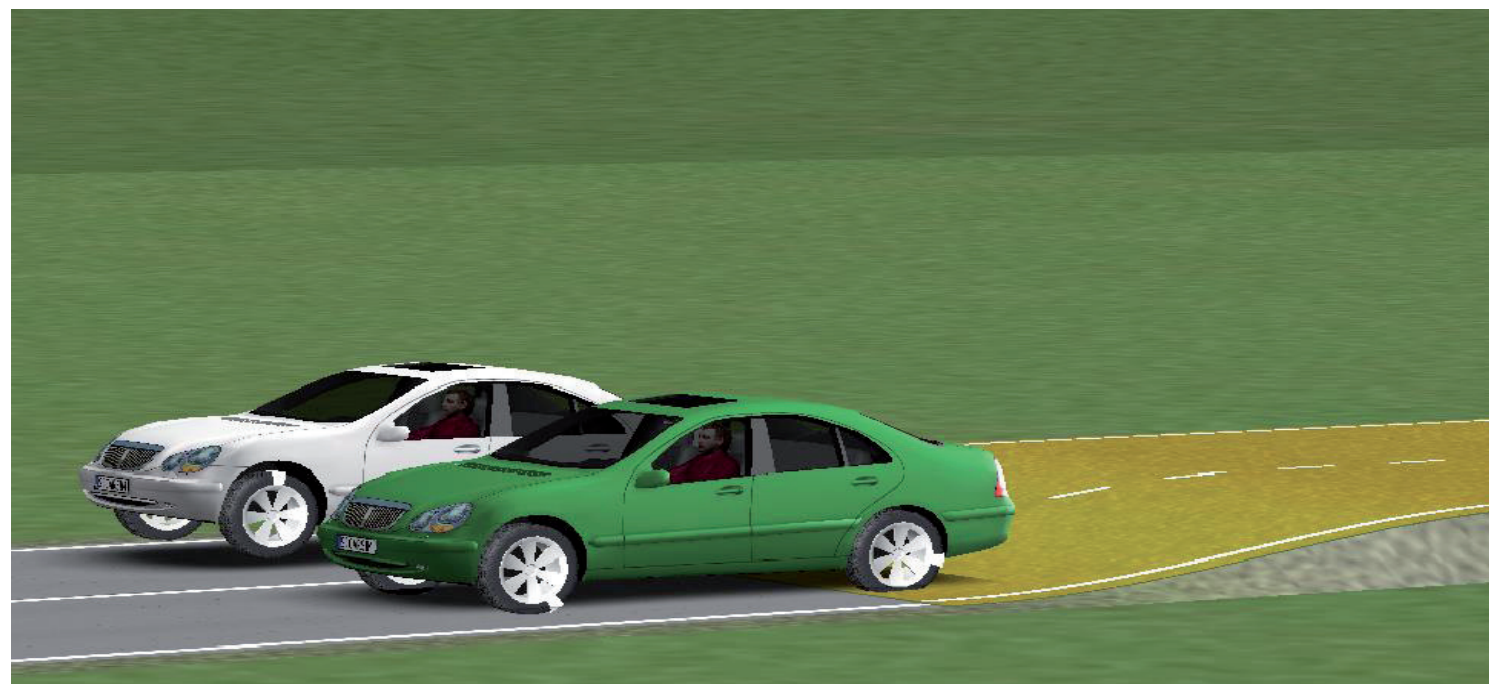

